\title{
Overview of left atrium phasic volumes using real time three dimensional echocardiography
}

\section{Abstract}

The reservoir, conduit, and contractile phasic volumes and functions of the left atrium (LA) are important to understand overall cardiac performance. As far as recent studies are concerned, the accurate assessment of LA phasic functions and structure, using techniques such as conventional two-dimensional echocardiography (2D-echo), real time three dimensional echocardiography (RT3DE), color tissue Doppler imaging (TDI), and speckle tracking echocardiography (STE), as well as cardiac computed tomography (CT) and magnetic resonance imaging (MRI). This review article explains how to determine the LA volume and functions using RT3DE and emphases their relationship with current diseases.

Keywords: left atrium, two-dimensional echocardiography, computed tomography, tissue doppler imaging
Volume I2 Issue I - 2019

\section{Batur Gonenc Kanar}

Department of Cardiology, Faculty of Medicine, Marmara University, Turkey

Correspondence: Batur Gonenc Kanar, Department of Cardiology, Faculty of Medicine, Marmara University, Turkey, Te +905395855150, Fax +902166570695, Email baturkanar@hotmail.com

Received: December II, 2018 | Published: January 04, 2019

\section{Introduction}

The importance of the LA in cardiovascular performance has long been acknowledged. The LA plays a pivotal role in stroke volume by modulating left ventricular (LV) filling with its reservoir, conduit, and contractile functions (Figure 1). ${ }^{1}$ The LA acts as a volume sensor of the diastolic burden, and communicates with the neurohormonal systems via the secretion of atrial natriuretic peptides. ${ }^{2}$ There is notable interplay between LA and LV function, such that events during each phase of "LA phasic function" are affected by factors from both the LA and LV.

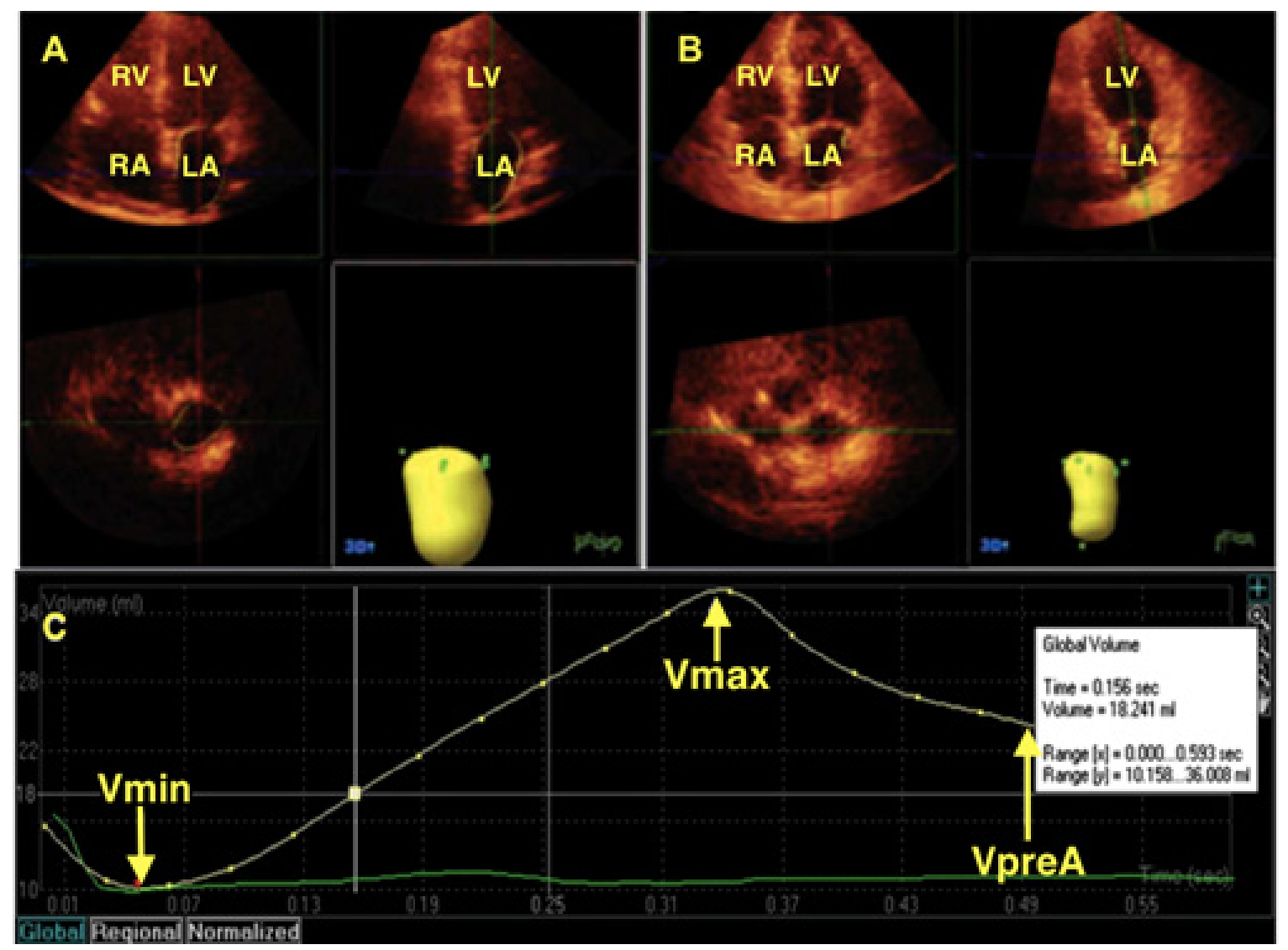

Figure I An example of left atrial volume quantification by real time three-dimensional echocardiography (RT3DE). Real time three-dimensional echocardiography recordings of max left atrial volume. (A) and min left atrial volume. (B) and time-volume curve. (C) indicating maximum and minimum left atrial volumes and before left atrial contraction volume. $\mathrm{V}_{\text {max }}$, maximum left atrial volume; $\mathrm{V}_{\text {min }}$, minimum left atrial volume; $\mathrm{V}_{\mathrm{pre}} \mathrm{A}$, left atrial volume before atrial contraction 


\section{Left atrium volume and measurements}

LA size increases with conditions of pressure overload, such as mitral stenosis and increased LV filling pressures, and conditions of volume overload, such as mitral regurgitation. LA size reflects the average effect of LV filling pressures over time, making it a useful marker of the severity of LV diastolic dysfunction. ${ }^{3}$ Measuring the maximum LA volume using conventional 2DE at the time of mitral valve opening is now routinely performed with calculation with a formula, but it only represents a snapshot of LA function at a specific point of the cardiac cycle and also its an assumption compare to RT3DE.4 In daily practice, prognostic information may be obtained by assessing LA phasic volumes (maximum LA volume [LA max], pre-atrial contraction LA volume [LA preA], and minimum LA volume [(LA min]) and deriving LA stroke volumes (Table 1). ${ }^{1}$

Table I Left atrium phasic volumes and functions

\begin{tabular}{lll}
\hline Reservoir function & Conduit function & Contractile function \\
\hline Total LA SV = LA max -LAmin & Passive LA SV = LA max -LA preA & Active LA SV = LA preA - LA min \\
Total LA EF = total LA SV/LAmax & Passive LA EF = passive LA SV/LAmax & Active LA EF = active LA SV/ LA pre A
\end{tabular}

$\mathrm{LA}_{\max }$, left atrium maksimum volume; LA ${ }_{\min }$, LA minimum volumme; LA preA, left atrium preatrial contraction volume; LA SV, left atrium stroke volume; LA EF, left atrium emptying fraction

In comparison with other current imaging modalities, the cardiac MRI and CT have higher spatial resolution than RT3DE. ${ }^{5,6}$ Another disadvantage of RT3DE is related with LA appendage. It has a considerable role for the function of LA. On account of shape of the LA appendage and the oblique position of the interatrial septum calculations may be resulted with errors in RT3DE. ${ }^{7}$ That is the reason why echocardiography systematically tends to underestimate LA volumes when compared to MRI or CT. On the other hand, RT3DE is more available and cost effective then the others in daily usage. ${ }^{8}$

\section{Clinical implication and current researches}

According to current researches, RT3DE is most suited to measuring phasic LA volumes among the other modalities. Because, it allows the assessment of individual LA function components and furthers our understanding of LA pathophysiology in various disease states. Kanar et al. ${ }^{9}$ measured LA phasic volumes and functions in hypertensive patients using RT3DE and revealed that LA phasic volumes and mechanical functions are associated with hypertensive end organ damaged. Moreover, the observed LA dilation in hypertensive patients (increased LA max, LA min, and LA preA volumes) was associated with augmentation of reservoir and conduit functions, but no significant change in atrial contractile function. In another RT3DE based study showed the relationship between LA phasic volumes and dysfunction with LV diastolic dysfunction and hypertensive retinopathy (according to the Keith-Wagener-Barker classification) in patients with systemic hypertension. The high grade hypertensive patients had higher LA phasic volumes and worse LV diastolic functions (higher E/e'). ${ }^{10}$ In a RT3DE LA volumes measured study, Atas et al. demonstrated that Patients with type 2 diabetes mellitus were found to have increased LA volumes and impaired atrial compliance and contractility. ${ }^{11}$ Eventually, increased LV filling pressure due to systemic hypertension, diabetes mellitus, or systemic diseases which can cause increased LV compliance might cause increase of LV phasic volumes and deterioration of the LA functions. Therefore, dedicated advanced LA imaging using RT3DE is mainly required in daily clinical practice. Currently, advanced in LA imaging may also provide new parameters with incremental prognostic information over existing techniques and ultimately improve management.

\section{Acknowledgment}

None.

\section{Funding}

None.

\section{Disclosure}

None.

\section{Conflicts of interest}

Author declares that there is no conflict of interest.

\section{References}

1. To AC, Flamm SD, Marwick TH, et al. Clinical utility of multimodality LA imaging: assessment of size, function and structure. $J A C C$ Cardiovasc Imaging. 2011;4(7):788-798.

2. Triposkiadis F, Pieske B, Butler J, et al. Global left in heart failure atrial failure. Eur J Heart Fail. 2016;18(11):1307-1320.

3. Tsang TS, Barnes ME, Gersh BJ, et al. Left atrial volume as a morphophysiologic expression of left ventricular diastolic dysfunction and relation to cardiovascular risk burden. Am J Cardiol. 2002;90(12):1284-1289.

4. Surkova E, Muraru D, Aruta P, et al. Current Clinical Applications of Three-Dimensional Echocardiography: When the Technique Makes the Difference. Curr Cardiol Rep. 2016;18(11):109.

5. Qureshi WT, Nasir UB. Principals and clinical applications of magnetic resonance cardiac spectroscopy in heart failure. Heart Fail Rev. 2017;22(4):491-499.

6. Bami K, Premaratne M, Lamba J, et al. Appropriate Use Criteria for Cardiac Computed Tomography: Impact on Diagnostic Utility. $J$ Comput Assist Tomogr. 2017;41(5):746-749.

7. Jenkins C, Chan J, Hanekom L, et al. Accuracy and feasibility of online 3-dimensional echocardiography for measurement of left ventricular parameters. J Am Soc Echocardiogr. 2006;19:1119-1128.

8. Lupu S, Mitre A, Dobreanu D. Left atrium function assessment by echocardiography - physiological and clinical implications. Med Ultrason. 2014;16:152-159.

9. Kanar B, Ozben B, Kanar HS, et al. Left atrial volume changes are an early marker of end-organ damage in essential hypertension: A multidisciplinary approach to an old problem. Echocardiography. 2017;34(12):1895-1902. 
10. Kanar BG, Kanar HS, Karatay A, et al. Assessment of left atrium and diastolic dysfunction in patients with hypertensive retinopathy: A real-time three-dimensional echocardiography-based study. Clin Exp Hypertens. 2017;39(8):696-704.
11. Atas H, Kepez A, Atas DB, et al. Effects of diabetes mellitus on left atrial volume and functions in normotensive patients without symptomatic cardiovascular disease. $J$ Diabetes Complications. 2014;28(6):858-862. 